\title{
Fatores ambientais associados ao sobrepeso infantil ${ }^{1}$
}

\author{
Environmental factors associated \\ with childhood overweight
}

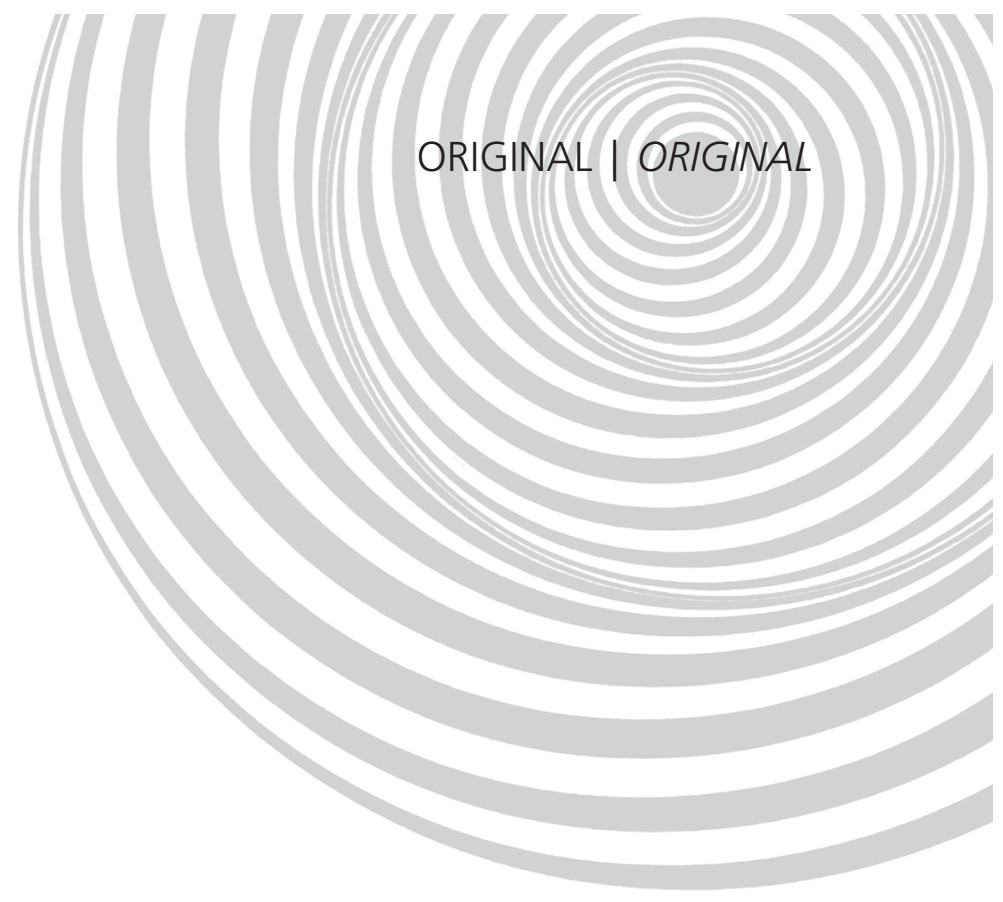

Juliana Farias de NOVAES²

Joel Alves LAMOUNIER ${ }^{3}$

Sylvia do Carmo Castro FRANCESCHINI ${ }^{4}$

Silvia Eloíza PRIORE ${ }^{4}$

RE S U M O

\section{Objetivo}

Avaliar os fatores ambientais intra-uterinos, perinatais e familiares associados ao sobrepeso infantil, ainda controversos na literatura.

\section{Métodos}

Estudo transversal realizado com 627 crianças, de 6 a 10 anos, matriculadas em escolas urbanas públicas e privadas do município de Viçosa (MG). O estado nutricional das crianças foi classificado pelo índice de massa corporal, segundo a preconização do Centers for Disease Control and Prevention. Foram aferidas as medidas de peso, estatura, pregas cutâneas tricipital e subescapular, circunferências da cintura e do quadril e pressão arterial das crianças, sendo os fatores ambientais obtidos por meio de questionário, segundo informações maternas. Classificou-se o estado nutricional das mães segundo a recomendação do World Health Organization.

\section{Resultados}

Do total de crianças, 87,9\% eram eutróficas e 12,1\% apresentavam sobrepeso. De acordo com a análise de regressão logística múltipla, os fatores associados ao sobrepeso infantil foram apresentar mãe obesa (OR: 6,92; $p<0,001)$, ser filho unigênito (OR: 1,87; $p=0,03$ ), permacer tempo superior a 3 horas diárias em frente à televisão (OR: 1,$91 ; p=0,04)$, não realizar educação física na escola (OR: 4,$80 ; p=0,02)$ e ser do sexo masculino (OR: 2,$60 ; p=0,001$ ).

\section{Conclusão}

Este estudo permite elucidar a identificação de fatores ambientais, potencialmente modificáveis, associados ao sobrepeso infantil no município de Viçosa. A determinação dos fatores ambientais é importante, uma vez

\footnotetext{
1 Artigo elaborado a partir da tese de J.F. NOVAES, intitulada "Fatores associados ao sobrepeso e à hipertensão arterial em escolares do município de Viçosa (MG). Universidade Federal de Minas Gerais; 2007. Apoio: Fundação de Amparo à Pesquisa do Estado de Minas Gerais (processo n CDS 1133/05).

2 Universidade Federal de Juiz de Fora, Departamento de Bioquímica. Campus Universitário, Martelos, 36036-900, Juiz de Fora, MG, Brasil. Correspondência para/Correspondence to: J.F. NOVAES. E-mail: <julianafnovaes@yahoo.com.br>.

${ }^{3}$ Universidade Federal de Minas Gerais, Faculdade de Medicina. Belo Horizonte, MG, Brasil.

${ }^{4}$ Universidade Federal de Viçosa, Departamento de Nutrição e Saúde. Viçosa, MG, Brasil.
} 
662 | J.F. NOVAES et al.

que o aumento na prevalência do sobrepeso na infância não pode ser totalmente explicado pelos fatores genéticos.

Termos de indexação: Características da família. Criança. Estudos transversais. Gestação. Sobrepeso.

\section{A B S T R A C T}

\section{Objective}

The objective of this study was to assess intrauterine, perinatal and family factors associated with childhood overweight, which are still controversial in the literature.

\section{Methods}

This cross-sectional study included a total of 627 children aged 6 to 10 years, who were enrolled in public and private schools in the city of Viçosa, Minas Gerais State. The nutritional status of the children was classified according to their body mass index, as recommended by the Centers for Disease Control and Prevention. The following data were collected: weight, height, tricipital and subscapular skinfold thicknesses, circumferences of the waist and hip, and blood pressure. The environmental factors were determined by means of a questionnaire, answered by the mother. The nutritional status of the mothers was classified according to the World Health Organization criteria.

\section{Results}

Out of 627 children, 551 were normal weight children and 76 were overweight. According to multiple logistic regression analysis, the following factors were associated with childhood overweight: maternal obesity (OR: 6.92; $p<0.001)$; being an only child (OR: 1.87; $p=0.03)$; watching television for more than 3 hours per day (OR: 1.91; $p=0.04)$; not practicing physical education at school (OR: 4.80; $p=0.02$ ) and being male (OR: 2.60; $p=0.001)$.

\section{Conclusion}

This study elucidated the potentially modifiable environmental factors associated with childhood overweight in the city of Viçosa. The determination of the environmental factors is important, since the increased prevalence of childhood overweight cannot be fully explained by genetic factors.

Indexing terms: Family characteristics. Child. Cross-sectional studies. Pregnancy. Overweight.

\section{N T R O D U Ç Ã O}

Muitos fatores ambientais já se apresentam claramente definidos como associados ao sobrepeso na infância, tais como hábitos alimentares indesejáveis, inatividade física e obesidade dos pais. No entanto, existem outros fatores que ainda precisam ser mais estudados, para melhor definir a relação causal com o sobrepeso infantil.

Estudos têm mostrado o tabagismo materno gestacional como fator associado ao sobrepeso infantil, conforme apresentado por Toschke et al. ${ }^{1}$ e von Kries et al. ${ }^{2}$. No primeiro, foi encontrada uma Odds Ratio (OR) de 1,92 (Intervalo de Confiança - IC=1,29-2,86) de obesidade, para aquelas crianças cujas mães fumaram durante sua respectiva gestação. No segundo, os autores constataram que a probabilidade de as crianças, que tiveram suas mães fumantes na gestação, apresentarem sobrepeso e obesidade foi de 1,43 $(I C=1,07-1,90)$ e 2,06 (IC=1,31-3,23), respectivamente. Fatores dietéticos maternos, associados ao tabagismo gestacional, podem ser uma justificativa para a relação observada entre o tabagismo materno e o sobrepeso infantil, pois, muitas muIheres fumam com o objetivo de perder peso e o fumo está associado à ingestão dietética reduzida². Ravelli et al. ${ }^{3}$ verificaram aumento na taxa de obesidade, em pessoas cuja nutrição foi deficiente na primeira metade da gravidez. Além desses fatores, tem sido documentado que a exposição fetal à nicotina no útero, em decorrência do tabagismo materno na gravidez, pode resultar em efeitos comportamentais persistentes, incluindo o 
déficit no controle do impulso alimentar². Uma possível deficiência no controle do impulso alimentar, em crianças expostas a produtos de tabaco na vida intra-uterina, pode resultar em redução da saciedade e aumento do consumo alimentar ${ }^{1}$.

Da mesma forma, autores têm observado uma relação significativa entre o alto peso ao nascer e o sobrepeso infantil ${ }^{4,5}$. É importante destacar que o peso ao nascer pode sofrer influência do estado nutricional materno na gestação, uma vez que a obesidade materna, neste período, pode favorecer o aumento de gordura corporal em recém-nascidos, sendo sugerido que o grau de adiposidade, ao nascimento, esteja correlacionado ao risco de obesidade, em etapas mais tardias ${ }^{6}$.

Além do tabagismo materno gestacional, do ganho excessivo de peso durante a gestação e da macrossomia das crianças, outros fatores ambientais relacionados ao sobrepeso infantil são tabagismo materno atual, número de pessoas no domicílio e número de irmãos da criança ${ }^{7-10}$. 0 tabagismo materno está, geralmente, associado a hábitos alimentares impróprios da mãe, que, certamente, podem influenciar os hábitos alimentares da criança. Além disso, o fato de pertencer à família com menor número de pessoas, ou ser filho único, pode representar um cuidado excessivo da mãe com a criança, tendendo a mesma ser mais preocupada com a alimentação de seu filho, o que pode resultar em ingestão energética excessiva, devido aos excessos de carinho, cuidado e zelo. Estudos também têm apresentado outros fatores, como associação do sobrepeso infantil à prematuridade, não amamentação ao seio e alta ou baixa escolaridade materna ${ }^{11,12}$.

É importante ressaltar que todos estes fatores ambientais ainda são muito controversos na literatura, existindo estudos que não encontraram nenhuma associação destes com o sobrepeso infantil ${ }^{13,14}$.

Diante do exposto, este estudo objetivou investigar fatores ambientais, que ainda precisam ser mais estudados para melhor definir a associação ao sobrepeso infantil, tais como características intra-uterinas (ganho de peso, tabagismo e idade gestacional); perinatais (peso ao nascer e aleitamento materno) e da família (escolaridade e tabagismo materno, número de irmãos da criança e número de pessoas residentes no domicílio), no município de Viçosa (MG).

\section{MÉ T O D O S}

Este estudo foi realizado no município de Viçosa, estado de Minas Gerais, Brasil. Este município é composto por, aproximadamente, 64854 habitantes, dos quais 59792 (92,2\%) residem na área urbana ${ }^{15}$.

Trata-se de um estudo transversal envolvendo escolares, com faixa etária de 6 a 10 anos, matriculados no ano de 2005 em 4 escolas urbanas do município de Viçosa (MG), sendo duas públicas e duas privadas. O critério de escolha dessas escolas foi o maior número de crianças matriculadas, incluindo, portanto, as duas maiores escolas públicas $(n=547$ e $n=258)$ e as duas maiores escolas privadas $(n=161$ e $n=210)$. Inicialmente, todos os 1176 escolares matriculados nessas escolas foram incluídos na amostra do estudo, tendo-se como critério de exclusão: aqueles que ainda não tinham completado 6 anos ou já haviam completado 11 anos ou mais ( $n=105) ;$ não tiveram a autorização dos pais para participar, seja pela falta de tempo destes $(n=37)$ ou pela justificativa de a criança já estar sob acompanhamento de outro profissional $(n=8)$; não localização dos responsáveis por telefone $(n=215)$; e recado não correspondido para os que não tinham telefone $(n=42)$. Assim, foram incluídas 769 crianças neste estudo, sendo 468 e 301 matriculadas em escolas públicas e privadas, respectivamente, representando $23,2 \%$ do total de crianças matriculadas em todas as escolas urbanas públicas (rede estadual) e privadas, no município de Viçosa, em 2005.

A avaliação antropométrica foi realizada em locais apropriados nas respectivas escolas, por um dos autores deste trabalho, a fim de minimizar 
possíveis vieses na aferição de medidas. Para obtenção do peso, utilizou-se balança portátil, digital e eletrônica, com capacidade de 150 quilos e sensibilidade de 50 gramas. A estatura foi verificada com estadiômetro, com extensão de 2 metros, dividido em centímetros e subdividido em milímetros. A aferição dessas duas medidas baseou-se na preconização de Jelliffe ${ }^{16}$. A partir dos valores de peso e estatura, calculou-se o Índice de Massa Corporal (IMC) e, de acordo com a idade e o sexo, definiu-se o estado nutricional das crianças segundo a classificação proposta pelo Centers for Disease Control and Prevention $(C D C)^{17}$. É importante ressaltar que, segundo o $\mathrm{CDC}^{17}$, sobrepeso é o termo preferido para referir crianças e adolescentes, cujo excesso de peso corporal pode resultar em riscos à saúde com conseqüências negativas. Valores de IMC superiores ao percentil 95 foram definidos como sobrepeso, e não obesidade, porque este índice não mede gordura corporal e não há consenso sobre seu ponto de corte, que indique obesidade em crianças ${ }^{18}$.

As pregas cutâneas tricipital e subescapular foram aferidas do lado direito do corpo, utilizando-se o equipamento Lange Skinfold Caliper. Cada medida foi verificada três vezes, não-consecutivas, sendo o resultado calculado pela média dos dois valores mais próximos. Para estimativa do percentual de gordura corporal das crianças, foram utilizadas as equações propostas por Slaughter et al. ${ }^{19}$, baseadas nos valores das pregas cutâneas tricipital e subescapular. Estas equações são derivadas de um modelo multicomponente e são ajustadas pelo sexo, grau de maturação e etnia. O grau de maturação considerado foi o pré-púbere e quanto à etnia, sendo as equações preditivas específicas para brancos e negros, as crianças morenas-claras e morenas-escuras tiveram o percentual de gordura estimado pelas equações preconizadas para a raça branca e negra, respectivamente. Foram considerados como adiposidade excessiva os valores de gordura corporal acima de $20 \%$ para meninos e de $25 \%$ para meninas ${ }^{20}$.

Utilizou-se fita métrica flexível e inelástica, para aferição das circunferências da cintura e quadril na posição intermediária entre o rebordo costal e a crista ilíaca, e na região da maior proeminência das nádegas, respectivamente ${ }^{21}$. A relação cintura/quadril foi obtida pela divisão entre o valor da circunferência da cintura e do quadril.

Os pais das crianças foram informados sobre os objetivos do trabalho e convidados a participar do estudo. Aqueles que aceitaram, espontaneamente, autorizaram a participação da criança, por assinatura do Termo de Consentimento Livre e Esclarecido. Posteriormente à avaliação antropométrica realizada na própria escola, com duração de 4 meses, aproximadamente, os pais foram convidados a agendar um horário para um atendimento nutricional com o profissional, para esclarecimento do resultado da avaliação antropométrica de seu filho e, quando necessário, para orientação quanto à reeducação alimentar e à alteração do estilo de vida da criança. Esse atendimento foi realizado no Laboratório de Avaliação Nutricional do Departamento de Nutrição e Saúde da Universidade Federal de Viçosa. Todas as medidas antropométricas da criança foram reavaliadas no atendimento indi-vidual, de forma a repassar, para os pais, os resul-tados mais atuais, sendo estes comparados com aqueles obtidos na avaliação, realizada na própria escola (aproximadamente aos 6 meses antes do atendimento individual). As crianças em distrofia nutricional (baixo peso, risco de sobrepeso e sobrepeso) foram acompanhadas, de forma a avaliar a melhora do estado nutricional ao longo do tempo.

Foram selecionadas as crianças com sobrepeso $(n=76)$ e as eutróficas ( $n=551$ ) para compor a amostra do estudo $(n=627)$. Durante $o$ atendimento nutricional, um questionário elaborado pelos autores foi aplicado principalmente às mães ou, na sua ausência, aos responsáveis pela criança. Esse questionário foi previamente testado com 30 mães de crianças de mesma faixa etária do estudo, não incluídas na amostra deste trabalho. O questionário englobou aspectos familiares, condições de gestação da mãe e do nascimento 
da criança, tempo de amamentação e prática de atividade física pela criança. Com a criança em repouso e deitada, foi avaliada a pressão sangüínea com um aparelho digital e eletrônico $\left(\mathrm{OMROM}^{\circledR}\right)$, por meio de uma braçadeira infantil, sendo a medida final calculada pelo valor médio de 3 aferições realizadas do lado direito do corpo, em intervalo de 5 minutos entre cada uma. A pressão arterial foi classificada, individualmente, segundo sexo, idade e percentil de estatura, de acordo com a preconização da Sociedade Brasileira de Hipertensão ${ }^{22}$, sendo considerados normotensão e hipertensão arterial os valores abaixo do percentil 90 e iguais ou superiores ao percentil 95, respectivamente.

As mães, cuja aceitação foi espontânea, tiveram seu estado nutricional avaliado pelo profissional, com aferição das medidas de peso e estatura. A partir dessas medidas, calculou-se o IMC, sendo este classificado de acordo com a World Health Organization $(\mathrm{WHO})^{18}$. Para melhor entendimento dos resultados, o termo pré-obeso foi substituído por sobrepeso, enquanto a discriminação das classes I, II e III da obesidade foram todas aglutinadas no termo único obesidade.

Quanto aos aspectos familiares, foi analisado o número total de pessoas que residiam no mesmo domicílio da criança, o número de irmãos desta e a escolaridade materna. Em relação aos aspectos da gestação materna e à condição de nascimento da criança, as mães foram interrogadas quanto ao tabagismo materno e ao ganho de peso gestacional, bem como sobre o peso ao nascer e a idade gestacional da criança.

O ganho de peso gestacional materno foi considerado excessivo, quando o valor era superior a $16 \mathrm{~kg}^{23}$. O peso ao nascer foi considerado baixo, insuficiente e normal para os valores entre $<2$ 500g, $2500 \mid-3000 \mathrm{~g}, \geq 3$ 000g, respectivamente ${ }^{24}$. Considerou-se como prematura, a termo e pós-termo, a criança cujo nascimento ocorreu antes de 37 semanas, entre a $37^{\mathrm{a}}$ e $41^{\mathrm{a}}$ semana e 6 dias, ou após 42 semanas, respectivamente ${ }^{24}$.
Foi questionado o tempo que a criança gastava, diariamente, em frente à televisão, ao videogame e ao computador, bem como as atividades físicas que realizava nos dias de semana, dentro e fora da escola.

Este estudo foi aprovado pelo Comitê de Ética em Pesquisa da Universidade Federal de Minas Gerais (Parecer n ETIC 0392/05).

O banco de dados e as análises estatísticas foram realizados com a utilização dos softwares Epi Info 6.025, Sigma-Stat $2.03^{26}$ e Statistical Program for Social Science (SPSS) 10.0 for Windows. As variáveis foram analisadas, primeiramente, como variáveis categóricas, e pelo teste do Qui-quadrado ou teste exato de Fisher, foram realizadas comparações bivariadas com a proporção de sobrepeso, sendo a Odds Ratio (OR) e o Intervalo de Confiança de 95\% calculados para cada variável. Para o ajuste das variáveis, foi utilizada a regressão logística múltipla, cujo ponto de corte definido para a inclusão destas foi $p$ inferior a 0,20 na análise bivariada com o sobrepeso, sendo esta a variável desfecho do estudo. As variáveis foram incluídas na análise de regressão pelo método enter, de acordo com o valor decrescente da Odds Ratio. Posteriormente, comparou-se a OR ajustada entre os dois grupos: sobrepeso (IMC/idade e sexo $\geq$ percentil 95) e eutrófico (IMC/idade e sexo < percentil 85).

Para verificar se a distribuição dos valores das variáveis era normal, utilizou-se o teste de normalidade de Kolmogorov-Smirnov. Conforme a distribuição das variáveis na curva normal, foram utilizados os testes $t$-Student e o de Mann-Whitney para comparação das médias e medianas entre dois grupos, respectivamente, bem como os testes de correlação de Pearson ou Spearman. Considerou-se, como nível de significância estatística, a probabilidade inferior a $5 \%(p<0,05)$.

\section{RESULTA DOS}

A proporção de sobrepeso e eutrofia na população foi $12,1 \%$ e $87,9 \%$, respectivamente. Na comparação das medidas antropométricas, de 
composição corporal e da pressão arterial entre as crianças com sobrepeso e eutróficas, observou-se que as primeiras apresentavam valores superiores para todas as medidas, com significância estatística $(p<0,001)$, de acordo com a Tabela 1.

Na análise bivariada, as variáveis que apresentaram associação estatística significante com o sobrepeso da criança foram: sexo $(p=0,004)$, faixa etária $(p=0,01)$, ordem do nascimento $(p=0,02)$, número de irmãos $(p=0,01)$, conforme a Tabela 2, atividades que costumam realizar nos dias de semana $(p=0,02)$ e nos fins de semana $(p=0,002)$, tempo por dia em frente à televisão $(p=0,004)$, realização de educação física na escola $(p=0,03)$, como mostra a Tabela 3, escolaridade materna atual $(p=0,01)$, ganho de peso gestacional $(p=0,01)$ e estado nutricional materno atual $(p<0,001)$, de acordo com a Tabela 4. Além destas variáveis, também foram incluídas na análise de regressão logística múltipla aquelas que apresentaram o valor de $p$ inferior a 0,20 na análise bivariada com o sobrepeso da criança, tais como se foi amamentado $(p=0,07)$, se reside com o pai e a mãe $(p=0,09)$, número de pessoas residentes no domicílio $(p=0,07)$, prática de atividade física fora da escola $(p=0,16)$ e tabagismo gestacional $(p=0,17)$, o que pode ser observado nas Tabelas 2,3 e 4 .

No modelo da regressão logística múltipla, verificou-se que os fatores ambientais, associados ao sobrepeso infantil, foram: obesidade materna $(p<0,001)$; criança unigênita $(p=0,03)$; tempo por dia em frente à televisão superior a 3 horas $(p=0,04)$; não realização de educação física na escola $(p=0,02)$; e sexo masculino $(p=0,001)$, segundo a Tabela 5 . As crianças cujas mães eram obesas apresentaram 6,92 vezes maior chance de sobrepeso, em comparação àquelas que não tinham mães obesas. Da mesma forma, crianças que eram unigênitas, passavam um tempo diário igual ou superior a 3 horas em frente à televisão ou não praticavam educação física na escola apresentaram 1,87; 1,91 e 4,80 vezes maior chance de ter sobrepeso, respectivamente, em relação àquelas que não apresentaram estas características. As variáveis de confusão, ou seja, aquelas que permaneceram associadas de maneira independente neste modelo, foram faixa etária, atividades que costumam realizar nos dias de semana e nos fins de semana, ordem do nascimento, número de pessoas que residem no domicílio, prática de atividade física fora da escola, ganho de peso e tabagismo gestacional materno (Tabela 5).

Tabela 1. Distribuição de crianças eutróficas e com sobrepeso, de 6 a 10 anos, segundo antropometria, composição corporal e pressão arterial. Viçosa (MG), 2005.

\begin{tabular}{|c|c|c|c|c|c|c|c|}
\hline \multirow{3}{*}{ Variáveis } & \multicolumn{6}{|c|}{ Crianças } & \multirow{3}{*}{$p$} \\
\hline & \multicolumn{3}{|c|}{ Sobrepeso } & \multicolumn{3}{|c|}{ Eutróficas } & \\
\hline & M & DP & Mi & M & DP & Mi & \\
\hline Peso $(\mathrm{kg})^{\mathbf{b}}$ & 49,5 & 10,8 & 50,1 & 29,6 & 5,8 & 28,4 & $<0,001^{*}$ \\
\hline Estatura $(\mathrm{cm})^{\mathrm{a}}$ & 141,5 & 9,4 & 141,2 & 134,0 & 9,3 & 133,6 & $<0,001^{*}$ \\
\hline Circunferência da cintura $(\mathrm{cm})^{\mathbf{b}}$ & 73,5 & 7,5 & 73,3 & 56,9 & 4,1 & 56,0 & $<0,001^{*}$ \\
\hline Circunferência do quadril $(\mathrm{cm})^{\mathbf{b}}$ & 87,1 & 8,8 & 88,5 & 68,9 & 6,0 & 68,3 & $<0,001^{*}$ \\
\hline Relação cintura/quadrilb & 0,8 & 0,05 & 0,8 & 0,8 & 0,05 & 0,8 & $<0,001^{*}$ \\
\hline Prega cutânea tricipital $(\mathrm{mm})^{\mathbf{b}}$ & 11,8 & 3,1 & 12,0 & 5,6 & 2,0 & 5,0 & $<0,001^{*}$ \\
\hline Prega cutânea subescapular $(\mathrm{mm})^{\mathbf{b}}$ & 14,7 & 4,6 & 14,0 & 6,0 & 1,9 & 5,5 & $<0,001^{*}$ \\
\hline Somatório de pregas cutâneas $(\mathrm{mm})^{\mathbf{b}}$ & 26,6 & 7,1 & 26,0 & 11,6 & 3,7 & 10,5 & $<0,001^{*}$ \\
\hline Gordura corporal $(\%)^{\mathbf{b}}$ & 23,7 & 5,3 & 23,6 & 10,9 & 3,7 & 10,1 & $<0,001^{*}$ \\
\hline Pressão arterial sistólica (mm Hg)a & 128,9 & 10,5 & 129,8 & 111,0 & 9,4 & 110,6 & $<0,001^{*}$ \\
\hline Pressão arterial diastólica $(\mathrm{mm} \mathrm{Hg})^{\mathbf{b}}$ & 81,0 & 10,0 & 80,3 & 67,7 & 6,9 & 67,3 & $<0,001^{*}$ \\
\hline
\end{tabular}

a variável com distribuição normal; b variável sem distribuição normal. * $p<0,05$. O valor de $p$ foi originado do correspondente teste $t$-Student ${ }^{a}$ ou teste de Mann-Whitney ${ }^{b}$.

M: média; DP: desvio-padrão; Mi: Mediana. 
Tabela 2. Proporção (\%) de sobrepeso e Odds Ratio (OR) bruta (Intervalo de Confiança - IC de 95\%), segundo as características das crianças de 6 a 10 anos. Viçosa (MG), 2005.

\begin{tabular}{|c|c|c|c|c|}
\hline Variáveis & Sobrepeso (\%) & Eutrofia (\%) & OR não ajustada (IC95\%) & $p$ \\
\hline \multicolumn{5}{|l|}{ Sexo } \\
\hline Feminino & 8,3 & 91,7 & 1,00 & \multirow[t]{2}{*}{$0,004^{*}$} \\
\hline Masculino & 15,7 & 84,3 & $2,06(1,21-3,53)$ & \\
\hline \multicolumn{5}{|l|}{ Faixa etária (anos) } \\
\hline $6-8$ & 8,3 & 91,7 & 1,00 & \multirow[t]{2}{*}{$0,01^{*}$} \\
\hline $9-10$ & 14,9 & 85,1 & $1,94(1,12-3,38)$ & \\
\hline \multicolumn{5}{|l|}{ Peso ao nascer (g) } \\
\hline$\geq 2500$ & 12,3 & 87,7 & 1,00 & \multirow[t]{2}{*}{0,60} \\
\hline$<2500$ & 9,8 & 90,2 & $0,78(0,26-2,13)$ & \\
\hline \multicolumn{5}{|l|}{ Idade gestacional } \\
\hline A termo e pós-termo & 11,6 & 88,4 & 1,00 & \multirow[t]{2}{*}{0,25} \\
\hline Pré-termo & 17,4 & 82,6 & $1,60(0,66-3,77)$ & \\
\hline \multicolumn{5}{|l|}{ Foi amamentado? } \\
\hline $\operatorname{sim}$ & 11,5 & 88,5 & 1,00 & \multirow[t]{2}{*}{0,07} \\
\hline Não & 20,9 & 79,1 & $2,03(0,86-4,64)$ & \\
\hline \multicolumn{5}{|l|}{ Ordem do nascimento } \\
\hline$\geq 2^{\circ}$ filho & 8,6 & 91,4 & 1,00 & \multirow[t]{2}{*}{$0,02^{*}$} \\
\hline $1^{\circ}$ filho & 14,8 & 85,2 & $1,84(1,08-3,16)$ & \\
\hline \multicolumn{5}{|l|}{ Número de irmãos } \\
\hline$\geq 1$ & 10,4 & 89,6 & 1,00 & \multirow[t]{2}{*}{$0,01^{*}$} \\
\hline Filho único & 18,7 & 81,3 & $1,98(1,13-3,47)$ & \\
\hline \multicolumn{5}{|l|}{ Tipo de escola } \\
\hline Pública & 11,7 & 88,3 & 1,00 & \multirow[t]{2}{*}{0,69} \\
\hline Privada & 12,7 & 87,3 & $1,10(0,66-1,84)$ & \\
\hline \multicolumn{5}{|l|}{ Mora com o pai e a mãe? } \\
\hline Com os dois ou só com a mãe & 11,7 & 88,3 & 1,00 & \multirow[t]{2}{*}{$0,09^{\mathrm{a}}$} \\
\hline Só com o pai ou nenhum dos dois & 22,2 & 77,8 & $2,16(0,75-5,91)$ & \\
\hline \multicolumn{5}{|l|}{ Número de pessoas no domicilio } \\
\hline$\geq 4$ & 10,9 & 89,1 & 1,00 & \multirow[t]{2}{*}{0,07} \\
\hline$<4$ & 16,5 & 83,5 & $1,63(0,92-2,85)$ & \\
\hline
\end{tabular}

*Significância estatística $(p<0,05)$; a $O$ valor de $p$ foi originado do teste Exato de Fisher e teste do Qui-Quadrado. Variáveis que apresentaram valores de $p$ em negrito foram incluídas na análise de regressão $(p<0,20)$.

\section{I S C U S S Ã O}

Neste estudo, maiores valores foram observados, nas crianças com sobrepeso, para as medidas de peso, altura, circunferência da cintura e do quadril, pregas cutâneas tricipital e subescapular, percentual de gordura corporal total e pressão arterial sistólica e diastólica. Este resultado confirma que crianças com sobrepeso já apresentam, pelo menos, um fator de risco cardiovascular, como distribuição central da gordura corporal e elevada pressão arterial, sendo importante ressaltar a possível existência de fatores genéticos.

A obesidade materna foi o fator ambiental mais fortemente associado ao sobrepeso infantil, no presente estudo. Resultados semelhantes foram encontrados por outros autores ${ }^{4,5,9,27-29}$.

Segundo Maffeis ${ }^{30}$, o mais forte fator de risco para obesidade infantil ainda é a obesidade dos pais, ocorrendo como um resultado do mapa genético, bem como das influências ambientais, 
668 | J.F. NOVAES et al.

Tabela 3. Proporção de sobrepeso (\%) e Odds Ratio (OR) bruta (Intervalo de Confiança - IC de 95\%), segundo a prática de atividade física das crianças de 6 a 10 anos. Viçosa (MG), 2005.

\begin{tabular}{|c|c|c|c|c|}
\hline Variáveis & Sobrepeso (\%) & Eutrofia (\%) & OR não ajustada (IC95\%) & $p$ \\
\hline \multicolumn{5}{|l|}{ O que costuma fazer em dias de semana? } \\
\hline $\begin{array}{l}\text { Bicicleta, patins, pega-pega, joga bola ou brinca de boneca, } \\
\text { casinha, carrinho ou desenha }\end{array}$ & 8,4 & 91,6 & 1,00 & $0,02^{*}$ \\
\hline Assiste televisão ou joga videogame & 14,8 & 85,2 & $1,89(1,09-3,30)$ & \\
\hline \multicolumn{5}{|l|}{ O que costuma fazer no fim de semana? } \\
\hline $\begin{array}{l}\text { Bicicleta, patins, pega-pega, joga bola ou brinca de boneca, } \\
\text { casinha, carrinho ou desenha }\end{array}$ & 9,8 & 90,2 & 1,00 & $0,002^{*}$ \\
\hline Assiste televisão ou joga videogame & 19,5 & 80,5 & $2,21(1,29-3,77)$ & \\
\hline \multicolumn{5}{|l|}{ Tempo por dia em frente à televisão (horas) } \\
\hline$\leq 2$ & 6,8 & 93,2 & 1,00 & $0,004^{*}$ \\
\hline$\geq 3$ & 14,7 & 85,3 & $2,36(1,24-4,53)$ & \\
\hline \multicolumn{5}{|l|}{ Faz educação física na escola? } \\
\hline Sim & 11,7 & 88,3 & 1,00 & $0,03^{\star a}$ \\
\hline Não & 36,4 & 63,6 & $4,31(1,03-16,91)$ & \\
\hline \multicolumn{5}{|l|}{ Faz atividade física fora da escola? } \\
\hline Sim & 15,0 & 85,0 & 1,00 & 0,16 \\
\hline Não & 10,9 & 89,1 & $0,70(0,41-1,19)$ & \\
\hline \multicolumn{5}{|l|}{ Freqüência da atividade física por semana } \\
\hline$\geq 3$ & 16,2 & 83,8 & 1,00 & 0,81 \\
\hline$\leq 2$ & 14,7 & 85,3 & $0,89(0,31-2,69)$ & \\
\hline
\end{tabular}

* Significância estatística $(p<0,05)$. O valor de $p$ foi originado do teste Exato de Fisher e teste do Qui-Quadrado.

Variáveis que apresentaram valores de $p$ em negrito foram incluídas na análise de regressão $(p<0,20)$.

Tabela 4. Proporção de sobrepeso (\%) e Odds Ratio (OR) bruta (Intervalo de Confiança - IC de 95\%), segundo as características das mães das crianças. Viçosa (MG), 2005.

\begin{tabular}{|c|c|c|c|c|}
\hline Variáveis & Sobrepeso (\%) & Eutrofia (\%) & OR não ajustada (IC95\%) & $p$ \\
\hline \multicolumn{5}{|l|}{ Escolaridade atual (anos) } \\
\hline$>13$ & 6,1 & 93,9 & 1,00 & $0,01^{*}$ \\
\hline$\leq 13$ & 13,9 & 86,1 & $2,47(1,15-5,46)$ & \\
\hline \multicolumn{5}{|l|}{ Ganho de peso gestacional } \\
\hline$\leq 16 \mathrm{~kg}$ & 10,8 & 89,2 & 1,00 & $0,01^{*}$ \\
\hline$>16 \mathrm{~kg}$ & 19,8 & 80,2 & $2,05(1,12-3,72)$ & \\
\hline \multicolumn{5}{|l|}{ Tabagismo gestacional } \\
\hline Não & 11,3 & 88,7 & 1,00 & 0,17 \\
\hline $\operatorname{sim}$ & 17,5 & 82,5 & $1,66(0,75-3,61)$ & \\
\hline \multicolumn{5}{|l|}{ Tabagismo atual } \\
\hline Não & 11,5 & 88,5 & 1,00 & 0,20 \\
\hline Sim & 16,5 & 83,5 & $1,51(0,76-2,95)$ & \\
\hline \multicolumn{5}{|l|}{ Estado nutricional atual } \\
\hline Baixo peso, eutrófico e sobrepeso & 8,4 & 91,6 & 1,00 & $<0,001$ \\
\hline Obesidade & 37,0 & 63,0 & $6,37(3,55-11,44)$ & \\
\hline \multicolumn{5}{|l|}{ Idade atual (anos) } \\
\hline $23-50$ & 11,9 & 88,1 & 1,00 & 0,43 \\
\hline$>50$ & 16,7 & 83,3 & $1,48(-)$ & \\
\hline
\end{tabular}

* Significância estatística $(p<0,05)$. O valor de $p$ foi originado do teste do Qui-Quadrado.

Variáveis que apresentaram valores de $p$ em negrito foram incluídas na análise de regressão $(p<0,20)$. 
Tabela 5. Odds Ratio (OR) bruta e ajustada dos fatores associados ao sobrepeso em crianças de 6 a 10 anos. Viçosa (MG), 2005.

\begin{tabular}{|c|c|c|c|c|c|}
\hline Variáveis & Sobrepeso (\%) & Eutrófico (\%) & $\begin{array}{l}\text { OR não ajustada } \\
\text { (IC 95\%) }\end{array}$ & $\begin{array}{l}\text { OR ajustada* } \\
\text { (IC 95\%) }\end{array}$ & $p$ \\
\hline Obesidade materna & 41,1 & 9,9 & $6,37(3,55-11,44)$ & $6,92(3,89-12,30)$ & $<0,001$ \\
\hline Criança unigênita & 31,6 & 18,9 & $1,98(1,13-3,47)$ & $1,87(1,05-3,34)$ & 0,030 \\
\hline Tempo/dia em frente à televisão ( $\geq 3 \mathrm{~h}$ ) & 81,6 & 65,3 & $2,36(1,24-4,53)$ & $1,91(1,01-3,63)$ & 0,040 \\
\hline Não pratica educação física na escola & 5,3 & 1,3 & $4,31(1,03-16,91)$ & $4,80(1,22-18,93)$ & 0,020 \\
\hline Sexo masculino & 67,1 & 49,7 & $2,06(1,21-3,53)$ & $2,60(1,49-4,52)$ & 0,001 \\
\hline
\end{tabular}

* Ajuste segundo características da criança (faixa etária, atividades que costumam realizar nos dias de semana e fins de semana, ordem do nascimento, número de pessoas que moram no domicílio, prática de atividade física fora da escola) e características maternas (ganho de peso e tabagismo gestacional).

entre elas o mesmo ambiente familiar. No entanto, a influência do estado nutricional dos pais no sobrepeso das crianças, em parte devido aos componentes genéticos, também é fortemente determinada pela ingestão alimentar da família, sendo importante ressaltar que o hábito alimentar materno tende a ser adotado pela criança, pois, geralmente, as mães são as mais envolvidas no preparo e na escolha dos alimentos ${ }^{9}$.

A criança não pode ser vista como uma unidade isolada, em que seu estado nutricional é solitariamente avaliado, sem perceber o indivíduo inserido em seu contexto familiar, interagindo com seu meio ambiente. A figura materna surge como importante elo entre criança-ambiente, estabelecendo-se uma íntima relação mãe e filho desde a gestação, evoluindo durante a infância, rumo à independência biológica e social da criança. No âmbito familiar, mãe e filhos compartilham condições socioambientais semelhantes, com hábitos alimentares associados, também aos aspectos culturais de cada grupo social, favorecendo uma relação direta em seu estado nutriciona ${ }^{27}$. A figura materna, geralmente, representa o elo entre criança e ambiente, além de ser a mãe quem normalmente decide sobre os hábitos alimentares da família, bem como os cuidados com a higiene e a imunização 29 .

O sucesso do tratamento da obesidade na infância inclui monitoramento próprio, mudança do comportamento alimentar e, principalmente, diminuição do sobrepeso nos pais, o que, provavelmente, contribuirá para a modificação positiva da alimentação e do estilo de vida da criança ${ }^{31}$. Segundo Mossberg ${ }^{32}$, a possibilidade de uma criança obesa continuar apresentando obesidade depende muito do nível de obesidade entre os membros da família, especialmente da mãe.

No presente estudo, observou-se que crianças unigênitas (filho único) apresentaram maior probabilidade de ter sobrepeso. É importante ressaltar que o efeito contribuinte do menor número de irmãos para o sobrepeso infantil pode ser a excessiva preocupação familiar, principalmente da mãe, mediante o cuidado com a criança, satisfazendo, mais facilmente, o desejo desta por guloseimas e estimulando-a a comer sempre mais que sua real necessidade, em virtude do cuidado excessivo. A associação entre o sobrepeso da criança e o fato de a mesma ser filho(a) único(a) foi, também, observada em outros estudos ${ }^{33-35}$. Toschke et al..$^{33}$ observaram que a presença de irmãos estava significativamente associada a uma baixa prevalência de obesidade em crianças. Oliveira et al. ${ }^{34}$, estudando crianças de 5 a 9 anos, constataram que o fato de a criança ser unigênita foi um fator preditivo independente para o desenvolvimento da obesidade $(\mathrm{OR}=1,5 ; \mathrm{IC}=1,2-1,8$; $p=0,02)$. Resultados semelhantes foram encontrados por Vignerová et al. ${ }^{35}$, que observaram maior risco de obesidade infantil entre famílias com, apenas, uma criança $(\mathrm{OR}=2,07 ; \mathrm{IC}=1,52-2,82$; $p<0,001)$. Além disso, o alto risco de obesidade entre crianças unigênitas pode ser explicado em razão de as famílias com apenas uma criança apresentarem melhor poder aquisitivo e, conseqüentemente, intensificarem a compra de ali- 
mentos preferidos e de maior densidade energética, em relação àquelas com mais de uma criança $^{10}$.

O tempo superior a 3 horas diárias em frente à televisão e a não realização de educação física na escola foram fatores associados ao sobrepeso infantil, no presente estudo. A associação existente entre o hábito de assistir televisão e o aumento na prevalência de obesidade em crianças ocorre, possivelmente, em função da natureza sedentária da atividade, acrescida da relação existente entre ela e o consumo de lanches baseados em alimentos ricos em gordura e açúcar, bem como pelo efeito cumulativo da exposição a propagandas de alimentos hipercalóricos ${ }^{34}$. As limitadas áreas de lazer devido à urbanização e as atrativas diversões em casa, como televisão, computador e videogame, pioram a situação ${ }^{7}$.

No presente estudo, verificou-se que a proporção do sobrepeso foi duas vezes superior no sexo masculino, em relação ao feminino $(67,1 \%$ vs $32,9 \%)$. Não se pode afirmar que ser do sexo masculino é um fator de risco para o sobrepeso infantil. Esta associação foi apenas uma característica da amostra. Talvez as crianças contempladas neste estudo necessitem de melhor orientação, quanto ao comportamento concernente aos hábitos alimentares e à prática de exercício físico, além da importância dos pais no sentido de garantir o consumo saudável de alimentos e a prática de atividade física regular. Resultados semelhantes foram verificados por Chen \& Kennedy ${ }^{36}$, que constataram que crianças chinesas de 8 a 10 anos, pertencentes ao sexo masculino, apresentaram maiores valores de IMC em relação às meninas, mesmo após o ajuste por variáveis de confusão. Além do sexo, a faixa etária mais elevada, o estilo de vida não rígido dos pais, o pouco controle dos pais sobre o comportamento da criança e a restrita comunicação com os pais foram fatores associados ao aumento do IMC.

Os fatores ambientais fortemente associados ao sobrepeso infantil são claramente comprovados na literatura: inatividade física e hábitos alimentares inadequados das crianças, além de obesidade materna. No presente estudo, o hábito alimentar das crianças não foi avaliado, pois em estudo prévio realizado em Viçosa, com crianças eutróficas e com sobrepeso de 6 a 8 anos, Novaes et al. ${ }^{37}$ observaram que os hábitos alimentares foram semelhantes entre os dois grupos, sendo observado maior tamanho das porções ingeridas pelas crianças com sobrepeso. Portanto, buscou-se investigar outros fatores ambientais, que precisam ser mais estudados, para melhor definir a associação ao sobrepeso infantil, tais como as características intra-uterinas (ganho de peso, tabagismo e idade gestacional), perinatais (peso ao nascer e aleitamento materno) e da família (escolaridade e tabagismo materno, número de irmãos da criança e número de pessoas residentes no domicílio). Os maiores valores de Odds Ratio, obtidos na análise de regressão logística multivariada, relacionavam-se à obesidade materna e ao sedentarismo da criança. Dentre as variáveis ambientais que, possivelmente, estão associadas ao sobrepeso infantil na literatura, somente o fato de a criança ser unigênita permaneceu estatisticamente significante pela análise de regressão no presente estudo.

Resultados semelhantes foram encontrados por Ochoa et al. ${ }^{14}$ que, estudando crianças e adolescentes de 6 a 18 anos na Espanha, observaram que os fatores relacionados ao estilo de vida atual estavam associados ao sobrepeso, tais como prática de atividade física, história familiar de obesidade, hábito de assistir televisão e consumo de refrigerantes. Fatores perinatais, como aleitamento materno e peso ao nascer, não estavam associados, sendo que, segundo os autores, parecem não exercer uma importante influência no desenvolvimento do sobrepeso infantil.

Muitos trabalhos têm sido publicados investigando os fatores de risco para o sobrepeso em crianças ${ }^{5,14,28}$. Nesses estudos, encontram-se diferentes fatores associados ao sobrepeso infantil, uma vez que cada um envolve diferentes populações, cada qual tendo seu próprio determinante geográfico, cultural e comportamental, sendo que 
a definição da obesidade também é diferente, devido à diversidade de critérios utilizados na classificação nutricional, com diferentes índices, pontos de corte e referências antropométricas. Diferenças metodológicas na classificação do sobrepeso, assim como diferenças nos padrões culturais e sociais dos estudos populacionais, provavelmente contribuem para achados inconsistentes entre os estudos.

Este estudo apresenta algumas limitações. Apesar de enfatizar a importância da investigação dos fatores ambientais associados ao sobrepeso infantil, a interpretação deve ser feita com cautela, uma vez que este trabalho baseia-se em delineamento transversal e, portanto, a denotação de causalidade deve ser interpretada com cuidado. Sua utilidade é gerar hipóteses, para que possam ser confirmadas, como inferências causais, por estudos longitudinais. Além disso, as associações descritas entre os vários fatores ambientais e o sobrepeso infantil podem ter sido confundidas por fatores não avaliados, tais como, ingestão dietética e nível socioeconômico. Como a amostra deste estudo foi socialmente heterogênea, a não avaliação da condição socioeconômica constitui uma limitação. Sendo uma característica importante associada ao sobrepeso, o nível socioeconômico deveria ter sido avaliado diretamente, por meio de questionamento relativo ao valor de renda mensal total ou per capita da família. No entanto, segundo Horta et al. ${ }^{38}$, é válido destacar a dificuldade em obter a informação, baseando-se na renda. Se essa informação não for precisa, o controle desta variável não assegurará completamente o efeito confundidor da renda verdadeira. Entretanto, o controle das variáveis de confusão, por meio da análise de regressão, constitui uma característica importante do estudo. Além disso, o fato de a coleta dos dados antropométricos e as características obtidas por meio de informações maternas terem sido exercidas somente por um pesquisador, contribui para reduzir os vieses de medida e de resposta.

Conclui-se que os fatores associados ao sobrepeso em crianças, no município de Viçosa, foram: obesidade materna; sexo masculino; criança unigênita; tempo superior a 3 horas diárias assistindo televisão; e a não realização de educação física na escola. A determinação dos fatores ambientais é importante, uma vez que o aumento na prevalência do sobrepeso na infância não pode ser totalmente explicado por meio de fatores genéticos. A identificação de fatores associados, potencialmente modificáveis, deve ser incorporada aos programas de prevenção e tratamento do excesso de peso infantil pelos profissionais de saúde. É importante a continuidade da investigação deste tema com trabalhos longitudinais, de forma a serem confirmadas as inferências causais entre fatores ambientais e sobrepeso infantil.

\section{AGRADECIMENTOS}

À Fundação de Amparo à Pesquisa do Estado de Minas Gerais, pela concessão da bolsa de doutorado e pelo financiamento do projeto (processo $n^{\circ}$ CDS 1133/05); ao Departamento de Nutrição e Saúde da Universidade Federal de Viçosa e à Faculdade de Medicina da Universidade Federal de Minas Gerais, pelo apoio; às crianças e aos pais, pela participação no estudo.

\section{COLABORADORES}

J.F. NOVAES contribuiu com a coleta de dados, a escrita do manuscrito e a análise estatística. J.A. LAMOUNIER, S.E. PRIORE e S.C.C. FRANCESCHINI contribuíram com a orientação do estudo e a revisão do manuscrito (redação e análise estatística).

\section{REFERÊ NCIAS}

1. Toschke AM, Koletzko B, Slikker Junior W, Hermann $\mathrm{M}$, von Kries RV. Childhood obesity is associated with maternal smoking in pregnancy. Eur J Pediatr. 2002; 161(8):445-8.

2. von Kries RV, Toschke AM, Koletzko B, Slikker Junior W. Maternal smoking during pregnancy and childhood obesity. Am J Epidemiol. 2002; 156(10): 954-61.

3. Ravelli GP, Stein ZA, Susser MW. Obesity in young men after famine exposure in utero and early infancy. N Engl J Med. 1976; 295(7):349-53. 
4. Kain J, Albala C, García F, Andrade M. Obesidad en el preescolar: evolución antropométrica y determinantes socioeconómicos. Rev Med Chilena 1998; 126(3):271-8.

5. Hui LL, Nelson EAS, Yu LM, Li AM, Fok TF. Risk factors for childhood overweight in 6- to 7-y-old Hong Kong children. Int J Obes. 2003; 27(11):1411-8.

6. Leibel RL, Hirsch J, Appel B, Checani GC. Energy intake required to maintain body weight is not affected by wide variation in diet composition. Am J Clin Nutr. 1992; 55(2):350-5.

7. Kagamimori S, Yamagami T, Sokejima S, Numata $\mathrm{N}$, Handa K, Nanri S, et al. The relationship between lifestyle, social characteristics and obesity in 3-yearold Japanese children. Child Care Health Dev. 1999; 25(3):235-47.

8. Gulliford MC, Mahabir D, Rocke B, Chinn S, Rona R. Overweight, obesity and skinfold thicknesses of children of African or Indian descent in Trinidad and Tobago. Int J Epidemiol. 2001; 30(5):989-98.

9. Marins VMR, Almeida RMVR, Pereira RA, Barros $M B A$. The relationship between parental nutritional status and overweight children/adolescents in Rio de Janeiro, Brazil. Public Health. 2004; 118(1): 43-9.

10. Maffeis C, Zaffanello M, Schutz Y. Relationship between physical inactivity and adiposity in prepubertal boys. J Pediatr. 1997; 131(2):288-92.

11. Laitinen J, Power C, Jarvelin M-R. Family social class, maternal body mass index, childhood body mass index, and age at menarche as a predictors of adult obesity. Am J Clin Nutr. 2001; 74(3):287-94.

12. Grummer-Strawn LM, Mei Z. Does breastfeeding protect against pediatric overweight? Analysis of longitudinal data from the Centers for Disease Control and Prevention Pediatric Nutrition Surveillance System. Pediatrics. 2004; 113(2):e81-e86.

13. Li L, Parsons TJ, Power C. Breast feeding and obesity in childhood: cross sectional study. BMJ. 2003; 327(7420):904-5.

14. Ochoa MC, Moreno-Aliaga MJ, Martínez-González MA, Martínez A, Marti A, GENOI Members. Predictor factors for childhood obesity in a Spanish case-control study. Nutrition. 2007; 23(5):379-84.

15. Instituto Brasileiro de Geografia e Estatística. Características da população e dos domicílios: resultados do universo. Censo demográfico. Rio de Janeiro: IBGE; 2000.

16. Jelliffe DB. Evaluación del estado de nutrición de la comunidad. Ginebra: OMS; 1968. OMS - Série de monografías, 53.

17. Centers for Disease Control and Prevention. CDC growth charts for the United States: methods and development. Vital Health Stat. 2000; 11(246): 1-190.

18. World Health Organization. Obesity: preventing and managing the global epidemic. Geneva: WHO; 1998. Report of a WHO Consultation on Obesity.

19. Slaughter MH, Lohman TG, Boileau RA, Horswill CA, Stillman RJ, van Loan MD, et al. Skinfold equations for estimation of body fatness in children and youth. Hum Biol. 1988; 60(5):709-23.

20. Lohman TG. Measuring body fat using skinfolds. Champaign (IL): Human Kinetics; 1987.

21. Taylor RW, Jones IE, Williams SM, Goulding A. Evaluation of waist circumference, waist-to-hip ratio, and the conicity index as screening tools for high trunk fat mass, as measured by dual-energy X-ray absorptiometry, in children aged 3-19y. Am J Clin Nutr. 2000; 72(2):490-5.

22. Sociedade Brasileira de Hipertensão. IV Diretrizes brasileiras de hipertensão arterial. Diagnóstico e classificação. Campos do Jordão; 2002.

23. Brasil. Ministério da Saúde. Secretaria Nacional de Programas Especiais de Saúde. Pré-natal de baixo risco. Brasília: Ministério da Saúde; 1988.

24. World Health Organization. Physical status: the use and interpretation of anthropometry. Geneva: WHO; 1995. Technical Report Series, 854.

25. Dean AG, Dean JA, Coulombier D, Brendel KA, Smith DC, Burton $A H$, et al. Epi Info: a word processing database, and statistics program for epidemiology on microcomputers, Version 6.0. Atlanta: CDCP; 1994.

26. Fox E, Kuo J, Tilling L, Ulrich C. User's manual-sigma stat: statistical software for Windows. Berlin: Jandel; 1994.

27. Engstrom EM, Anjos LA. Relação entre o estado nutricional materno e sobrepeso nas crianças brasileiras. Rev Saúde Pública. 1996; 30(3):233-9.

28. He Q, Ding ZY, Fong DYT, Karlberg J. Risk factors of obesity in preschool children in China: a population-based case-control study. Int J Obes. 2000; 24(11):1528-36.

29. Drachler ML, MacLuf SPZ, Leite JCC, Aerts DRGC, Giugliani ERJ, Horta BL. Fatores de risco para sobrepeso em crianças no sul do Brasil. Cad Saúde Pública. 2003; 19(4):1073-81.

30. Maffeis C, Zantedeschi P, Filippi L, Matti P, Boscarol G, Grezzani A, et al. Patterns of food intake and obesity in Italian children. Int J Obes. 1999; 23(Suppl 5):S44.

31. Epstein LH, McCurley J, Wing RR, Valoski A. Five-year follow-up of family-based behavioral 
treatments for childhood obesity. J Consult Clin Psychol. 1990; 58(5):661-4.

32. Mossberg H-O. 40-year follow-up of overweight children. Lancet. 1989; 26(8661):491-3.

33. Toschke AM, Vignerova J, Lhotska L, Osancova K, Kolestzko B, von Kries R. Overweight and obesity in 6- to 14-year-old Czech children in 1991: protective effect of breast-feeding. J Pediatr. 2002; 141(6):764-9.

34. Oliveira AMA, Cerqueira EMM, Souza JS, Oliveira AC. Sobrepeso e obesidade infantil: influência de fatores biológicos e ambientais em Feira de Santana, BA. Arq Bras Endocrinol Metab. 2003; 47(2):144-50.

35. Vignerová J, Bláha P, Osancová K, Roth Z. Social inequality and obesity in Czech school children. Econ Hum Biol. 2004; 2(1):107-18.
36. Chen J-L, Kennedy C. Family functioning, parenting style, and Chinese children's weight status. J Fam Nurs. 2004; 10(2):262-79.

37. Novaes JF, Franceschini SCC, Priore SE. Hábitos alimentares de crianças eutróficas e com sobrepeso em Viçosa, MG, Brasil. Rev Nutr. 2007; 20(6): 633-42. doi: 10.1590/\$1415-52732007000600 006.

38. Horta BL, Bahl R, Martines JC, Victora CG. Evidence on the long-term effects of breastfeeding: sytematic reviews and meta-analyses. Geneve: WHO; 2007.

Recebido em: 23/10/2007

Versão final reapresentada em: 22/8/2008

Aprovado em: 15/5/2009 